\title{
RIEMANN INTEGRAL OF A RANDOM FUNCTION AND THE PARABOLIC EQUATION WITH A GENERAL STOCHASTIC MEASURE
}

UDC 519.21

\author{
V. RADCHENKO
}

\begin{abstract}
For a stochastic parabolic equation driven by a general stochastic measure, the weak solution is obtained. The integral of a random function in the equation is considered as a limit in probability of Riemann integral sums. Basic properties of such integrals are studied in this paper.
\end{abstract}

\section{INTRODUCTION}

In this paper we consider the stochastic parabolic equation, which can be formally written as

$$
d X(x, t)=A X(x, t) d t+f(x, t) d \mu(t), \quad X(x, 0)=\xi(x),
$$

where $(x, t) \in \mathbb{R}^{d} \times[0, T], A$ is a second-order strongly elliptic differential operator, and $\mu$ is a general stochastic measure defined on the Borel $\sigma$-algebra of $[0, T]$. For $\mu$ we assume $\sigma$-additivity in probability only; assumptions for $A, f$ and $\xi$ are given in Section 6 . Equation (1.1) is interpreted in the weak sense (see (6.1) below). We prove the existence and uniqueness of the solution.

The weak form of (1.1) includes the integral of a random function with respect to deterministic measure (Jordan content). We interpret this integral as a limit in the probability of Riemann integral sums. This definition of the integral allows us to interchange the order of integration with respect to deterministic and stochastic measures (Theorem 4.1) that is important for solving the equation. A large part of the paper is devoted to the study of this Riemann-type stochastic integral.

Parabolic stochastic partial differential equations (SPDEs) driven by the martingale measures had been introduced and discussed initially in [19]. This approach was later developed in [1, 3. Parabolic SPDEs as equations in infinite dimensional space were studied in [4, 11]. In these and many other papers the stochastic noise has some distributional, integrability or martingale properties. In our paper, we consider a very general class of possible $\mu$ on $[0, T]$. On the other hand, the stochastic term in (1.1) is independent of $u$. One reason is that the appropriate definition of an integral of a random function with respect to $\mu$ does not exist.

Some motivating examples for studying SPDEs may be found in [4, Introduction] and [6. section 13.2]. For $A=\Delta$, (1.1) describes the evolution in time of the density $X$

2000 Mathematics Subject Classification. Primary 60H05, 60H15.

Key words and phrases. Stochastic measure, Riemann integral, stochastic parabolic equation, weak solution.

This research was supported by Alexander von Humboldt Foundation, grant no. UKR/1074615. The author wishes to thank Professor M. Zähle for fruitful discussions, and the hospitality of Jena University is gratefully acknowledged. 
of some quantity for such a heat or chemical concentration in a system with random sources. In our model, the random influence can be rather general.

\section{Preliminaries}

Let $L_{0}=L_{0}(\Omega, \mathcal{F}, \mathrm{P})$ be a set of all real-valued random variables defined on a complete probability space $(\Omega, \mathcal{F}, \mathrm{P})$ (or equivalence classes of). Convergence in $L_{0}$ means the convergence in probability and is the convergence in the quasi-norm

$$
\|\eta\|=\inf \{\delta: \operatorname{P}\{|\eta|>\delta\} \leq \delta\} .
$$

Note that $\left\|\eta_{1}+\eta_{2}\right\| \leq\left\|\eta_{1}\right\|+\left\|\eta_{2}\right\|$. The following inequality will be used in the sequel:

$$
\left\|\sum_{k=1}^{l} c_{k} \xi_{k}\right\| \leq 8 \max _{a_{k}= \pm 1}\left\|\sum_{k=1}^{l} a_{k} \xi_{k}\right\| \leq 16 \max _{V}\left\|\sum_{k \in V} \xi_{k}\right\|, \quad\left|c_{k}\right| \leq 1, \xi_{k} \in L_{0},
$$

where the latter maximum is taken over all possible $V \subset\{1, \ldots, l\}$ (see [16. Theorem 3]).

Let $\mathrm{S}$ be an arbitrary set and $\mathcal{B}$ be a $\sigma$-algebra of subsets of $\mathrm{S}$.

Definition 2.1. Any $\sigma$-additive mapping $\mu: \mathcal{B} \rightarrow L_{0}$ is called a stochastic measure.

In other words, $\mu$ is a vector measure with values in $L_{0}$. We do not assume positivity or integrability for stochastic measures. In [7] such a $\mu$ is called a general stochastic measure. In the following, $\mu$ always denotes a stochastic measure.

Examples of stochastic measures are the following. Let $S=[0, T] \subset \mathbb{R}_{+}, \mathcal{B}$ be the $\sigma$-algebra of Borel subsets of $[0, T]$, and $Y(t)$ be a square integrable martingale. Then $\mu(\mathrm{A})=\int_{0}^{T} \mathbf{1}_{\mathrm{A}}(t) d Y(t)$ is a stochastic measure. If $W^{H}(t)$ is a fractional Brownian motion with Hurst index $H>\frac{1}{2}$ and $f:[0, T] \rightarrow \mathbb{R}$ is a bounded measurable function, then $\mu(\mathrm{A})=\int_{0}^{T} f(t) \mathbf{1}_{\mathrm{A}}(t) d W^{H}(t)$ is also a stochastic measure, as follows from [8, Theorem 1.1]. Some other examples may be found in [7, subsection 7.2]. Theorem 8.3.1 in [7] states the conditions under which the increments of a real-valued Lévy process generates a stochastic measure.

For deterministic measurable functions $g: \mathrm{S} \rightarrow \mathbb{R}$, an integral of the form $\int_{\mathrm{S}} g d \mu$ is studied in [12] (see also [7, Chapter 7] and [2]). The construction of this integral is standard, uses an approximation by simple functions and is based on results of [15, 17, 18, In particular, every bounded measurable $g$ is integrable with respect to any $\mu$. An analogue of the Lebesgue dominated convergence theorem holds for this integral (see [7, Proposition 7.1.1] or [12, Corollary 1.2]).

For equations with stochastic measures, weak solutions of some SPDEs were obtained in [13. Regularity properties of a mild solution of the stochastic heat equation were considered in 14.

\section{Riemann integral of A RANDOM Function}

Let $\mathrm{B} \subset \mathbb{R}^{d}$ be a Jordan measurable set and $\xi: \mathrm{B} \rightarrow L_{0}$ be a random function. We shall say that $\xi$ has an integral on $B$ if for any sequence of partitions

$$
\mathrm{B}=\bigcup_{1 \leq k \leq k_{n}} \mathrm{~B}_{k n}, \quad n \geq 1, \quad \max _{k} \operatorname{diam} \mathrm{B}_{k n} \rightarrow 0, \quad n \rightarrow \infty, \quad x_{k n} \in \mathrm{B}_{k n},
$$

the limit in probability

$$
\mathrm{p} \lim _{n \rightarrow \infty} \sum_{1 \leq k \leq k_{n}} \xi\left(x_{k n}\right) \mathrm{m}\left(\mathrm{B}_{k n}\right)=\int_{\mathrm{B}} \xi(x) d x
$$

exists. Here $\mathrm{m}$ denotes the Jordan content, and the sets $\mathrm{B}_{k n}, 1 \leq k \leq k_{n}$, are assumed to be Jordan measurable and have no common interior points. By the mixing of different 
sequences of partitions, we can prove that the limit is independent of the choice of the sequence. For deterministic $\xi$, our definition is equivalent to the definition of the standard Riemann integral in [9].

Lemma 3.1. Let $\xi$ be an integral on $\mathrm{B}=\prod_{k=1}^{d}\left[a_{k}, b_{k}\right] \subset \mathbb{R}^{d}$. Then the set of values $\{\xi(x), x \in \mathrm{B}\}$ is bounded in probability.

Proof. Is analogous to the deterministic case.

For some other $\mathrm{B} \subset \mathbb{R}^{d}$, limit (3.1) can exist for unbounded $\xi$ (for instance, in the case $m(B)=0)$. We use the following

Definition 3.1. The random function $\xi$ is called integrable on $\mathrm{B}$ if $\xi$ has an integral on $\mathrm{B}$ and set of values $\{\xi(x), x \in \mathrm{B}\}$ is bounded in probability.

Let $\tilde{B} \subset \mathbb{R}^{d}$ be an unbounded set for which there exists a sequence of Jordan measurable sets $\mathrm{B}^{(j)}$ such that

$$
\mathrm{B}^{(j)} \uparrow \tilde{\mathrm{B}}, \quad \forall c>0 \exists j: \tilde{\mathrm{B}} \cap\{|x| \leq c\} \subset \mathrm{B}^{(j)}
$$

(we call $\mathrm{B}^{(j)}$ the exhaustive set). We shall say that $\xi$ is integrable (in an improper sense) on $\tilde{B}$ if $\xi$ is integrable on each $\mathrm{B}^{(j)}$, and there exists the limit in probability

$$
\mathrm{p} \lim _{j \rightarrow \infty} \int_{\mathrm{B}^{(j)}} \xi(x) d x=\int_{\tilde{\mathrm{B}}} \xi(x) d x
$$

that is independent of the choice of $\mathrm{B}^{(j)}$.

All bounded subsets of $\mathbb{R}^{d}$ used in this paper are assumed to be Jordan measurable, and all unbounded sets are assumed to be approximable by Jordan measurable sets in the sense of (3.2). Sets in partitions are assumed to be non-overlapping.

Obviously, if $\xi$ has the Riemann integrable paths, then $\xi$ is integrable in our sense. Theorem 4.1 below gives other examples of integrable random functions.

Further, we establish basic properties of the integral.

Lemma 3.2. Let $\xi$ be integrable on $\mathrm{B}$. Then $\xi$ is integrable on each $\mathrm{A} \subset \mathrm{B}$, and for any $\varepsilon>0$ there exists $\delta>0$ such that for all $\mathrm{A} \subset \mathrm{B}, \mathrm{A}=\bigcup_{1 \leq k \leq k_{0}} \mathrm{~A}_{k}, x_{k} \in \mathrm{A}_{k}$, and $\operatorname{diam} \mathrm{A}_{k}<\delta$, there holds

$$
\left\|\sum_{1 \leq k \leq k_{0}} \xi\left(x_{k}\right) \mathrm{m}\left(\mathrm{A}_{k}\right)-\int_{\mathrm{A}} \xi(x) d x\right\|<\varepsilon .
$$

Proof. Suppose the lemma were false. Then

$$
\begin{gathered}
\exists \varepsilon_{0}>0 \forall \delta>0 \exists \mathrm{A}=\bigcup_{1 \leq k \leq k_{0}} \mathrm{~A}_{k}=\bigcup_{1 \leq i \leq i_{0}} \mathrm{~A}_{i}^{\prime}, \operatorname{diam} \mathrm{A}_{k}, \operatorname{diam} \mathrm{A}_{i}^{\prime}<\delta: \\
\left\|\sum_{1 \leq k \leq k_{0}} \xi\left(x_{k}\right) \mathrm{m}\left(\mathrm{A}_{k}\right)-\sum_{1 \leq i \leq i_{0}} \xi\left(x_{i}^{\prime}\right) \mathrm{m}\left(\mathrm{A}_{i}^{\prime}\right)\right\| \geq \varepsilon_{0} .
\end{gathered}
$$

Take an arbitrary partition

and add

$$
\mathrm{B} \backslash \mathrm{A}=\bigcup_{1 \leq j \leq j_{0}} \mathrm{C}_{j}, \quad \operatorname{diam} \mathrm{C}_{j}<\delta
$$

$$
\sum_{1 \leq j \leq j_{0}} \xi\left(x_{j}^{\prime \prime}\right) \mathrm{m}\left(\mathrm{C}_{j}\right), \quad x_{j}^{\prime \prime} \in \mathrm{C}_{j},
$$

to each of the considered sums on A. Thus we can get two integral sums on B with arbitrarily small diameters such that the quasi-norm of their difference is greater than or equal to $\varepsilon_{0}$. This contradicts the integrability of $\xi$ on $B$. 
Lemma 3.3. Let $\xi$ be integrable on $\tilde{\mathrm{B}}$ in the improper sense, $\tilde{\mathrm{A}} \subset \tilde{\mathrm{B}}$. Then $\xi$ is integrable on $\tilde{\mathrm{A}}$ (if $\tilde{\mathrm{A}}$ is an unbounded set, the integral is meant in the improper sense).

Proof. Take the exhaustive sets $\mathrm{B}^{(j)} \uparrow \tilde{\mathrm{B}}, \mathrm{A}^{(i)} \uparrow \tilde{\mathrm{A}}$. Then the sets

$$
\left(\mathrm{B}^{(j)} \backslash \tilde{\mathrm{A}}\right) \cup \mathrm{A}^{(i)} \uparrow \tilde{\mathrm{B}}, \quad i, j \rightarrow \infty
$$

are exhaustive too, and

$$
\int_{\tilde{\mathrm{B}}} \xi(x) d x=\mathrm{p} \lim _{i, j \rightarrow \infty}\left(\int_{\mathrm{B}^{(j)} \backslash \tilde{\mathrm{A}}} \xi(x) d x+\int_{\mathrm{A}^{(i)}} \xi(x) d x\right) .
$$

If $\mathrm{p} \lim _{i \rightarrow \infty} \int_{\mathrm{A}^{(i)}} \xi(x) d x$ does not exist, then we can choose $i, j \rightarrow \infty$ such that the limit in (3.3) does not exist.

Lemma 3.4. Let $\xi$ be integrable on $\mathrm{B}$. Then the set of values $\left\{\int_{\mathrm{A}} \xi(s) d s, \mathrm{~A} \subset \mathrm{B}\right\}$ is bounded in probability.

Proof. Suppose the lemma were false. Then

$$
\exists \varepsilon_{0}>0, \mathrm{~A}_{n} \subset \mathrm{B}, n \geq 1: \quad\left\|\frac{1}{n} \int_{\mathrm{A}_{n}} \xi(s) d s\right\| \geq \varepsilon_{0} .
$$

By Lemma [3.2, we can choose a partition $\mathrm{B}=\bigcup_{1 \leq k \leq k_{0}} \mathrm{~B}_{k}$ fine enough such that all integral sums for partitions $\mathrm{A}_{n}=\bigcup_{1 \leq k \leq k_{0}}\left(\mathrm{~A}_{n} \cap \mathrm{B}_{k}\right)$ will be close enough to the integrals on $\mathrm{A}_{n}$. Thus, for all $n, x_{k n} \in \mathrm{A}_{n} \cap \mathrm{B}_{k}$, we get

$$
\left\|\frac{1}{n} \sum_{1 \leq k \leq k_{0}} \xi\left(x_{k n}\right) \mathrm{m}\left(\mathrm{A}_{n} \cap \mathrm{B}_{k}\right)\right\| \geq \frac{\varepsilon_{0}}{2} .
$$

Since the number of summands is fixed for all $n$, we arrive at a contradiction with the boundedness of $\xi$.

Lemma 3.5. Let $\xi$ be integrable on $\mathrm{B}$, and $f: \mathrm{B} \rightarrow \mathbb{R}$ be a deterministic uniformly continuous function on $\mathrm{B}$. Then $f \xi$ is integrable on $\mathrm{B}$.

Proof. Consider the difference of two integral sums of $f \xi$ :

$$
\begin{aligned}
& \left\|\sum_{1 \leq k \leq k_{m}} f\left(x_{k m}\right) \xi\left(x_{k m}\right) \mathrm{m}\left(\mathrm{B}_{k m}\right)-\sum_{1 \leq i \leq i_{n}} f\left(x_{i n}\right) \xi\left(x_{i n}\right) \mathrm{m}\left(\mathrm{B}_{i n}\right)\right\| \\
& =\left\|\sum_{1 \leq k \leq k_{m}, 1 \leq i \leq i_{n}}\left[f\left(x_{k m}\right) \xi\left(x_{k m}\right)-f\left(x_{i n}\right) \xi\left(x_{i n}\right)\right] \mathrm{m}\left(\mathrm{B}_{k m} \cap \mathrm{B}_{i n}\right)\right\| \\
& \leq\left\|\sum_{1 \leq k \leq k_{m}, 1 \leq i \leq i_{n}}\left[\xi\left(x_{k m}\right)-\xi\left(x_{i n}\right)\right] f\left(x_{i n}\right) \mathrm{m}\left(\mathrm{B}_{k m} \cap \mathrm{B}_{i n}\right)\right\| \\
& \quad+\left\|\sum_{1 \leq k \leq k_{m}, 1 \leq i \leq i_{n}}\left[f\left(x_{k m}\right)-f\left(x_{i n}\right)\right] \xi\left(x_{k m}\right) \mathrm{m}\left(\mathrm{B}_{k m} \cap \mathrm{B}_{i n}\right)\right\| \\
& =S_{1}+S_{2} .
\end{aligned}
$$

From (2.1), for $|f(x)| \leq C$ we get

$$
S_{1} \leq 16 \max _{V}\left\|C \sum_{(k, i) \in V}\left[\xi\left(x_{k m}\right)-\xi\left(x_{i n}\right)\right] \mathrm{m}\left(\mathrm{B}_{k m} \cap \mathrm{B}_{i n}\right)\right\|,
$$

where the maximum is taken over all possible sets of pairs $(k, i)$. 
For example, consider

$$
\begin{aligned}
\sum_{(k, i) \in V} & \xi\left(x_{k m}\right) \mathrm{m}\left(\mathrm{B}_{k m} \cap \mathrm{B}_{i n}\right) \\
= & \sum_{1 \leq k \leq k_{m}} \xi\left(x_{k m}\right)\left[\sum_{i:(k, i) \in V} \mathrm{~m}\left(\mathrm{~B}_{k m} \cap \mathrm{B}_{i n}\right)+\mathrm{m}\left(\mathrm{B}_{k m} \cap \mathrm{B}_{i^{\prime} n}\right) \mathbf{1}_{x_{k m} \notin\left(\cup_{i:(k, i) \in V} \mathrm{~B}_{i n}\right)}\right) \\
& -\sum_{1 \leq k \leq k_{m}} \xi\left(x_{k m}\right) \mathrm{m}\left(\mathrm{B}_{k m} \cap \mathrm{B}_{i^{\prime} n}\right) \mathbf{1}_{x_{k m} \notin\left(\bigcup_{i:(k, i) \in V} \mathrm{~B}_{i n}\right)} \\
= & I_{1}-I_{2} .
\end{aligned}
$$

Here $\mathrm{B}_{i^{\prime} n}$ is one of the sets $\mathrm{B}_{i n}, 1 \leq i \leq i_{n}$, that contains $x_{k m}$. (If $x_{k m}$ lies on the border of $\mathrm{B}_{i^{\prime} n}$, we take it only once.) $I_{1}$ and $I_{2}$ are integral sums and, by Lemma 3.2. they approximate the integrals of $\xi$ on respective sets. Therefore, for a diameter small enough, $I_{1}-I_{2}$ will be close to the integral on $\bigcup_{(k, i) \in V}\left(\mathrm{~B}_{k m} \cap \mathrm{B}_{i n}\right)$. Similarly,

$$
\sum_{(k, i) \in V} \xi\left(x_{i n}\right) \mathrm{m}\left(\mathrm{B}_{k m} \cap \mathrm{B}_{i n}\right)
$$

approximates the integral on the same set, and we make the right hand side of (3.4) arbitrarily small by choosing the diameter.

Further, for any $\alpha>0$, for diameter small enough and $\mathrm{B}_{k m} \cap \mathrm{B}_{\text {in }}=\varnothing$, we have $\left|f\left(x_{k m}\right)-f\left(x_{i n}\right)\right|<\alpha$ in $S_{2}$. Inequality (2.1) implies

$$
S_{2} \leq 16 \max _{V}\left\|\alpha \sum_{(k, i) \in V} \xi\left(x_{k m}\right) \mathrm{m}\left(\mathrm{B}_{k m} \cap \mathrm{B}_{i n}\right)\right\| .
$$

As before, we can make the sum arbitrarily close to the integral on $\bigcup_{(k, i) \in V}\left(\mathrm{~B}_{k m} \cap \mathrm{B}_{i n}\right)$. From Lemma 3.4 it follows that $S_{2} \rightarrow 0$ as $\alpha \rightarrow 0$.

Lemma 3.6. Let $\xi$ be integrable on $\mathrm{B}$, and $f: \mathrm{B} \rightarrow \mathbb{R}$ be a deterministic uniformly continuous function on $\mathrm{B},|f(x)| \leq C$. Then

$$
\left\|\int_{\mathrm{B}} f(x) \xi(x) d x\right\| \leq 16 \sup _{\mathrm{A} \subset \mathrm{B}}\left\|C \int_{\mathrm{A}} \xi(x) d x\right\| .
$$

Proof. The inequality for respective integral sums follows from (2.1). Further, we pass to the limit and apply Lemmas 3.2 and 3.5 .

Lemma 3.7. Let $\xi$ be integrable on $\mathrm{B}, f_{n}: \mathrm{B} \rightarrow \mathbb{R}, n \geq 1$, be a deterministic uniformly continuous functions on $\mathrm{B}$, and $\sup _{x \in \mathrm{B}}\left|f_{n}(x)\right| \rightarrow 0, n \rightarrow \infty$. Then

$$
\int_{\mathrm{B}} f_{n}(x) \xi(x) d x \stackrel{\mathrm{P}}{\rightarrow} 0, \quad n \rightarrow \infty .
$$

Proof. The statement follows from Lemmas 3.4 and 3.6 .

Lemma 3.8. Let $\xi$ be integrable on an unbounded set $\tilde{\mathrm{B}}$ in an improper sense, and $f: \tilde{\mathrm{B}} \rightarrow \mathbb{R}$ be a deterministic bounded uniformly continuous function on $\tilde{\mathrm{B}}$. Then $f \xi$ is integrable on $\tilde{\mathrm{B}}$ in an improper sense.

Proof. For $\mathrm{B}^{(j)} \uparrow \tilde{\mathrm{B}}$ and $|f(x)| \leq C$ Lemma 3.6 implies

$$
\left\|\int_{\mathrm{B}^{(j)} \backslash \mathrm{B}^{(i)}} f(x) \xi(x) d x\right\| \leq 16 \sup _{\mathrm{A} \subset\left(\mathrm{B}^{(j)} \backslash \mathrm{B}^{(i)}\right)}\left\|C \int_{\mathrm{A}} \xi(x) d x\right\| .
$$

If the left hand side of (3.5) does not tend to 0 as $i, j \rightarrow \infty$, then we can construct a sequence of bounded sets $\mathrm{C}^{(j)} \uparrow \tilde{\mathrm{B}}$ such that the sequence $\int_{\mathrm{C}_{j}} \xi(x) d x, j \geq 1$, is nonfundamental. 
Lemma 3.9. Let $\xi$ be integrable on an unbounded set $\tilde{\mathrm{B}}$ in an improper sense, and $f_{n}: \tilde{\mathrm{B}} \rightarrow \mathbb{R}$ be a deterministic bounded uniformly continuous on $\tilde{\mathrm{B}}$ functions,

$$
\sup _{n \geq 1, x \in \tilde{\mathrm{B}}}\left|f_{n}(x)\right|=C<\infty, \quad \sup _{x \in \mathrm{B}}\left|f_{n}(x)\right| \rightarrow 0, \quad n \rightarrow \infty,
$$

for all bounded $\mathrm{B} \subset \tilde{\mathrm{B}}$. Then

$$
\int_{\tilde{\mathrm{B}}} f_{n}(x) \xi(x) d x \stackrel{\mathrm{P}}{\rightarrow} 0, \quad n \rightarrow \infty .
$$

Proof. Suppose the lemma is false. Applying Lemma 3.7, one can find $\varepsilon_{0}>0$, a subsequence $f_{n_{j}}, j \geq 1$, and a bounded disjoint sets $\mathrm{B}_{j} \subset(\tilde{\mathrm{B}} \cap\{|x| \geq j\})$ such that

$$
\left\|\int_{\mathrm{B}_{j}} f_{n_{j}}(x) \xi(x) d x\right\|>\varepsilon_{0} .
$$

From Lemma 3.6 it follows that there exist bounded disjoint sets $\mathrm{A}_{j} \subset(\tilde{\mathrm{B}} \cap\{|x| \geq j\})$ such that $\left\|C \int_{\mathrm{A}_{j}} \xi(x) d x\right\|>\left(\varepsilon_{0} / 16\right)$. This contradicts the integrability of $\xi$ on $\tilde{\mathrm{B}}$.

Note that the stochastic continuity of $\xi$ does not imply the integrability.

Example 3.1. Consider $\mathrm{B}=[0,1]$ and $\xi_{k}(\omega)=5^{k} \mathbf{1}_{F_{k}}, k \geq 1$, where $\mathrm{P}\left(F_{k}\right)=1 / k$, with $F_{k}$ independent. Set

$$
\begin{cases}\xi(0)=0, & \\ \xi(x)=\xi_{k}, & 2^{-2 k-1} \leq x \leq 2^{-2 k} \\ \xi(x)=2^{2 k+2}\left(\left(2^{-2 k-1}-x\right) \xi_{k+1}+\left(x-2^{-2 k-2}\right) \xi_{k}\right), & 2^{-2 k-2} \leq x \leq 2^{-2 k-1} .\end{cases}
$$

Taking all possible finite unions $\mathrm{A}=\bigcup_{k}\left[2^{-2 k-1}, 2^{-2 k}\right]$, we see that the values $\int_{\mathrm{A}} \xi(x) d x$ are not bounded in probability. By Lemma 3.4 $\xi$ is not integrable on $[0,1]$.

\section{INTERCHANGE OF THE ORDER OF INTEGRATION}

Theorem 4.1. Let $\mu$ be a stochastic measure on $(\mathrm{S}, \mathcal{B})$, and $\mathrm{B} \subset \mathbb{R}^{d}$ be a bounded set. Assume that $h(x, s): \mathrm{B} \times \mathrm{S} \rightarrow \mathbb{R}$ is a measurable deterministic function which is Riemann integrable on $\mathrm{B}$ for each fixed $s$, and $|h(x, s)| \leq g(s)$, where $g: \mathbf{S} \rightarrow \mathbb{R}$ is integrable on $\mathbf{S}$ with respect to $d \mu(s)$. Then the random function $\xi(x)=\int_{\mathrm{S}} h(x, s) d \mu(s)$ is integrable on $\mathrm{B}$, and

$$
\int_{\mathrm{B}} d x \int_{\mathrm{S}} h(x, s) d \mu(s)=\int_{\mathrm{S}} d \mu(s) \int_{\mathrm{B}} h(x, s) d x .
$$

Proof. From the inequality $|h(x, s)| \leq g(s)$ and (2.1) it follows that the values of $\xi$ are bounded in probability (see Lemma 1.1 and Theorem 1.3 in [12]). Integral sums of $\int_{\mathrm{B}} \xi(x) d x$ have the form

$$
\begin{gathered}
\sum_{1 \leq k \leq k_{n}} \mathrm{~m}\left(\mathrm{~B}_{k n}\right) \int_{\mathrm{S}} h\left(x_{k n}, s\right) d \mu(s)=\int_{\mathrm{S}} g_{n}(s) d \mu(s), \\
g_{n}(s)=\sum_{1 \leq k \leq k_{n}} h\left(x_{k n}, s\right) \mathrm{m}\left(\mathrm{B}_{k n}\right) \rightarrow \int_{\mathrm{B}} h(x, s) d x .
\end{gathered}
$$

The boundedness condition of $h$ and the analogue of the Lebesgue theorem [7, Proposition 7.1.1] for the integral with respect to $d \mu(s)$ imply the statement. 
Corollary 4.1. Let $\mu$ be a stochastic measure on $(\mathrm{S}, \mathcal{B})$, and $\tilde{\mathrm{B}} \subset \mathbb{R}^{d}$ be an unbounded set. Assume that $h(x, s): \tilde{\mathrm{B}} \times \mathrm{S} \rightarrow \mathbb{R}$ is a measurable deterministic function which is Riemann integrable on $\tilde{\mathrm{B}}$ in an improper sense for each fixed $s$, and $|h(x, s)| \leq g(s)$,

$$
\int_{\tilde{\mathrm{B}}}|h(x, s)| d x=g_{1}(s)
$$

where $g, g_{1}: \mathrm{S} \rightarrow \mathbb{R}$ are integrable on $\mathbf{S}$ with respect to $d \mu(s)$. Then the random function $\xi(x)=\int_{\mathrm{S}} h(x, s) d \mu(s)$ is integrable on $\tilde{\mathrm{B}}$ in an improper sense, and

$$
\int_{\tilde{\mathrm{B}}} d x \int_{\mathrm{S}} h(x, s) d \mu(s)=\int_{\mathrm{S}} d \mu(s) \int_{\tilde{\mathrm{B}}} h(x, s) d x .
$$

Proof. For bounded sets $\mathrm{B}^{(j)} \uparrow \tilde{\mathrm{B}}$, Theorem 4.1 implies

$$
\int_{\mathrm{B}^{(j)}} d x \int_{\mathrm{S}} h(x, s) d \mu(s)=\int_{\mathrm{S}} d \mu(s) \int_{\mathrm{B}^{(j)}} h(x, s) d x .
$$

Further, we use the analogue of the Lebesgue theorem and the integrability of $g_{1}$.

Theorem 4.2. Let $\mathrm{B} \subset \mathbb{R}^{d}, \mathrm{~S} \subset \mathbb{R}^{m}$ be bounded sets, and the random function

$$
\xi(x, s): \mathrm{B} \times \mathrm{S} \rightarrow L_{0}
$$

be integrable on $\mathrm{B} \times \mathrm{S}$ with respect to $d x \times d s$ and be integrable on $\mathrm{S}$ with respect to $d s$ for each fixed $x$. Then

$$
\int_{\mathrm{B} \times \mathrm{S}} \xi(x, s) d x \times d s=\int_{\mathrm{B}} d x \int_{\mathrm{S}} \xi(x, s) d s .
$$

Proof. The integral sums for the integral with respect to $d x$ in (4.3) has the form

$$
\sum_{1 \leq k \leq k_{0}} \mathrm{~m}\left(\mathrm{~B}_{k}\right) \int_{\mathrm{S}} \xi\left(x_{k}, s\right) d s
$$

Each integral in (4.4) may be approximated by sums of the form $\sum_{1 \leq i \leq i_{0}} \mathrm{~m}\left(\mathrm{~S}_{i}\right) \xi\left(x_{k}, s_{i}\right)$. Thus, the sums

$$
\sum_{1 \leq k \leq k_{0}} \sum_{1 \leq i \leq i_{0}} \mathrm{~m}\left(\mathrm{~B}_{k}\right) \mathrm{m}\left(\mathrm{S}_{i}\right) \xi\left(x_{k}, s_{i}\right)
$$

will approximate the right hand side of (4.4). But they are the integral sums for the integral with respect to $d x \times d s$ in (4.3), and will be close to the left hand side of (4.3) for sufficiently small diameters of $\mathrm{B}_{k} \times \mathrm{S}_{i}$.

Corollary 4.2. Let $\mathrm{S} \subset \mathbb{R}^{m}$ be a bounded set, and $\tilde{\mathrm{B}} \subset \mathbb{R}^{d}$ be an unbounded set. Assume that the random function $\xi(x, s): \tilde{\mathrm{B}} \times \mathrm{S} \rightarrow L_{0}$ is integrable on $\tilde{\mathrm{B}} \times \mathrm{S}$ with respect to $d x \times d s$ in an improper sense, is integrable on $\tilde{\mathrm{B}}$ with respect to $d x$ in an improper sense for each fixed $s$, and is integrable on $\mathrm{S}$ with respect to $d s$ for each fixed $x$. Then

$$
\int_{\tilde{\mathrm{B}} \times \mathrm{S}} \xi(x, s) d x \times d s=\int_{\mathrm{S}} d s \int_{\tilde{\mathrm{B}}} \xi(x, s) d x=\int_{\tilde{\mathrm{B}}} d x \int_{\mathrm{S}} \xi(x, s) d s .
$$

Proof. Consider exhaustive sets $\mathrm{B}^{(j)} \uparrow \tilde{\mathrm{B}}$. For the first of the repeated integrals (4.5), the integral sums have the form

$$
\sum_{1 \leq i \leq i_{0}} \mathrm{~m}\left(\mathrm{~S}_{i}\right) \int_{\tilde{\mathrm{B}}} \xi\left(x, s_{i}\right) d x .
$$

The integrals in (4.6) can be approximated by $\int_{\mathrm{B}^{(j)}} \xi\left(x, s_{i}\right) d x$, and the last integral is the limit of the sums

$$
\sum_{1 \leq k \leq k_{0}} \mathrm{~m}\left(\mathrm{~B}_{k}^{(j)}\right) \xi\left(x_{k}^{(j)}, s_{i}\right) \text {. }
$$


If the integral sums in (4.6) do not converge, then we can construct a non-convergent sequence of sums

$$
\sum_{1 \leq i \leq i_{0}} \sum_{1 \leq k \leq k_{0}} \mathrm{~m}\left(\mathrm{~S}_{i}\right) \mathrm{m}\left(\mathrm{B}_{k}^{(j)}\right) \xi\left(x_{k}^{(j)}, s_{i}\right),
$$

and this contradicts the integrability of $\xi$ on $\mathrm{S} \times \tilde{\mathrm{B}}$.

Further, by Theorem 4.2 for each $j$ we have

$$
\int_{\mathrm{B}^{(j)} \times \mathrm{S}} \xi(x, s) d x \times d s=\int_{\mathrm{B}^{(j)}} d x \int_{\mathrm{S}} \xi(x, s) d s .
$$

The left hand side has the limit in probability as $j \rightarrow \infty$. Hence, the right hand side has the limit, and the second equality of (4.5) holds.

\section{INTEGRATION BY PARTS}

To solve the parabolic stochastic equation, we need the following two lemmas.

Lemma 5.1. Let a random function $\xi(u):[0, s] \rightarrow L_{0}$ be integrable on $[0, s]$. Then $\eta(u)=\int_{0}^{u} \xi(v) d v$ is integrable on $[0, s]$, and

$$
\int_{0}^{s} d u \int_{0}^{u} \xi(v) d v=\int_{0}^{s}(s-v) \xi(v) d v
$$

Proof. By Lemma 3.5 the function $(s-v) \xi(v)$ is integrable, and by Lemma $3.2 \eta(u)$ is well defined. The integral sum of $\int_{0}^{s} \eta(u) d u$ has the form

$$
\sum_{1 \leq k \leq k_{0}} \mathrm{~m}\left(\mathrm{~B}_{k}\right) \int_{0}^{u_{k}} \xi(v) d v, \quad u_{k} \in \mathrm{B}_{k}
$$

We can take a new partition $[0, s]=\bigcup_{1 \leq i \leq i_{0}} \mathrm{C}_{i}$ such that each integral $\int_{0}^{u_{k}} \xi(v) d v$ can be close enough to th integral sum with this partition (Lemma 3.2). Thus we can approximate (5.1) arbitrarily close by the sum

$$
\sum_{1 \leq k \leq k_{0}} \mathrm{~m}\left(\mathrm{~B}_{k}\right) \sum_{1 \leq i \leq i_{0}} \mathrm{~m}\left(\mathrm{C}_{i} \cap\left[0, u_{k}\right]\right) \xi\left(v_{i}\right), \quad v_{i} \in \mathrm{C}_{i} .
$$

For $\int_{0}^{s}(s-v) \xi(v) d v$, take the integral sum

$$
\sum_{1 \leq i \leq i_{0}} \mathrm{~m}\left(\mathrm{C}_{i}\right)\left(s-v_{i}\right) \xi\left(v_{i}\right)
$$

The difference of (5.3) and (5.2) is equal to

$$
\sum_{1 \leq i \leq i_{0}} \xi\left(v_{i}\right)\left[\mathrm{m}\left(\mathrm{C}_{i}\right)\left(s-v_{i}\right)-\mathrm{m}\left(\mathrm{C}_{i}\right) \sum_{k: \mathrm{C}_{i}<\mathrm{B}_{k}} \mathrm{~m}\left(\mathrm{~B}_{k}\right)-\sum_{k: \mathrm{C}_{i} \cap \mathrm{B}_{k} \neq \varnothing} \mathrm{m}\left(\mathrm{B}_{k}\right) \mathrm{m}\left(\mathrm{C}_{i} \cap\left[0, u_{k}\right]\right)\right] .
$$

The notation $\mathrm{C}_{i}<\mathrm{B}_{k}$ means that $v<u$ for all $v \in \mathrm{C}_{i}, u \in \mathrm{B}_{k}$. We have

$$
0 \leq\left(s-v_{i}\right)-\sum_{k: \mathrm{C}_{i}<\mathrm{B}_{k}} \mathrm{~m}\left(\mathrm{~B}_{k}\right) \leq \max _{i} \operatorname{diam} \mathrm{C}_{i}+\max _{k} \operatorname{diam} \mathrm{B}_{k} .
$$

The last sum of (5.4) is not greater than

$$
\mathrm{m}\left(\mathrm{C}_{i}\right) \sum_{k: \mathrm{C}_{i} \cap \mathrm{B}_{k} \neq \varnothing} \mathrm{m}\left(\mathrm{B}_{k}\right) \leq \mathrm{m}\left(\mathrm{C}_{i}\right)\left(\max _{i} \operatorname{diam} \mathrm{C}_{i}+2 \max _{k} \operatorname{diam} \mathrm{B}_{k}\right) .
$$

Therefore, value (5.4) may be written in the form $\sum_{1 \leq i \leq i_{0}} \xi\left(v_{i}\right) \mathrm{m}\left(\mathrm{C}_{i}\right) \alpha_{i}$, where $\alpha_{i} \rightarrow 0$ as $\operatorname{diam} \mathrm{C}_{i}$, diam $\mathrm{B}_{k} \rightarrow 0$. From (2.1) we obtain

$$
\left\|\sum_{1 \leq i \leq i_{0}} \xi\left(v_{i}\right) \mathrm{m}\left(\mathrm{C}_{i}\right) \alpha_{i}\right\| \leq 16 \max _{V}\left\|\max _{i}\left|\alpha_{i}\right| \sum_{i \in V} \xi\left(v_{i}\right) \mathrm{m}\left(\mathrm{C}_{i}\right)\right\| .
$$


The sums $\sum_{i \in V} \xi\left(v_{i}\right) \mathrm{m}\left(\mathrm{C}_{i}\right)$ are close to respective integrals for diam $\mathrm{C}_{i}$ small enough (Lemma 3.2) and the values of integrals are bounded in probability (Lemma 3.4). Therefore, the left hand side of (5.5) tends to zero as $\max _{i}\left|\alpha_{i}\right| \rightarrow 0$.

Lemma 5.2. Let a random function $\xi(u):[0, s] \rightarrow L_{0}$ be integrable on $[0, s]$, and

$$
f \in \mathbb{C}^{(1)}([0, s])
$$

be a deterministic function. Then

$$
f(s) \int_{0}^{s} \xi(u) d u=\int_{0}^{s} f(u) \xi(u) d u+\int_{0}^{s} f^{\prime}(u) d u \int_{0}^{u} \xi(v) d v .
$$

Proof. From Lemmas 3.5 and 5.1 it follows that the random functions

$$
\zeta_{1}(u)=f(u) \xi(u), \quad \zeta_{2}(u)=f^{\prime}(u) \int_{0}^{u} \xi(v) d v
$$

are integrable on $[0, s]$. First, let us show that for

$$
0=u_{0}<u_{1}<\cdots<u_{k_{0}}=s, \quad \alpha=\max _{k}\left|u_{k}-u_{k-1}\right|,
$$

we have

$$
\sum_{1 \leq k \leq k_{0}}\left(f\left(u_{k}\right)-f\left(u_{k-1}\right)\right) \int_{0}^{u_{k}} \xi(v) d v \stackrel{\mathrm{P}}{\rightarrow} \int_{0}^{s} f^{\prime}(u) d u \int_{0}^{u} \xi(v) d v, \quad \alpha \rightarrow 0 .
$$

Applying the Lagrange formula and integrability of $\zeta_{2}$, for some $\tilde{u}_{k} \in\left(u_{k-1}, u_{k}\right)$ we obtain

$$
\begin{gathered}
\sum_{1 \leq k \leq k_{0}}\left(f\left(u_{k}\right)-f\left(u_{k-1}\right)\right) \int_{0}^{u_{k}} \xi(v) d v=\sum_{1 \leq k \leq k_{0}} f^{\prime}\left(\tilde{u}_{k}\right)\left(u_{k}-u_{k-1}\right) \int_{0}^{u_{k}} \xi(v) d v \\
=\sum_{1 \leq k \leq k_{0}} f^{\prime}\left(\tilde{u}_{k}\right)\left(u_{k}-u_{k-1}\right) \int_{0}^{\tilde{u}_{k}} \xi(v) d v+\sum_{1 \leq k \leq k_{0}} f^{\prime}\left(\tilde{u}_{k}\right)\left(u_{k}-u_{k-1}\right) \int_{\tilde{u}_{k}}^{u_{k}} \xi(v) d v, \\
\sum_{1 \leq k \leq k_{0}} f^{\prime}\left(\tilde{u}_{k}\right)\left(u_{k}-u_{k-1}\right) \int_{0}^{\tilde{u}_{k}} \xi(v) d v \stackrel{\mathrm{P}}{\rightarrow} \int_{0}^{s} f^{\prime}(u) d u \int_{0}^{u} \xi(v) d v, \quad \alpha \rightarrow 0 .
\end{gathered}
$$

For $C_{1}=\max _{u}\left|f^{\prime}(u)\right|$, from (2.1) we have

$$
\begin{aligned}
& \left\|\sum_{1 \leq k \leq k_{0}} f^{\prime}\left(\tilde{u}_{k}\right)\left(u_{k}-u_{k-1}\right) \int_{\tilde{u}_{k}}^{u_{k}} \xi(v) d v\right\| \\
& \quad \leq 16 \max _{V}\left\|C_{1} \alpha \sum_{k \in V} \int_{\tilde{u}_{k}}^{u_{k}} \xi(v) d v\right\| \leq 16 \sup _{\mathrm{A}}\left\|C_{1} \alpha \int_{\mathrm{A}} \xi(v) d v\right\| .
\end{aligned}
$$

From Lemma 3.4 it follows that the last value tends to 0 as $\alpha \rightarrow 0$. Therefore, (5.7) is proved.

Integrability of $\zeta_{1}$ implies

$$
\begin{aligned}
& \sum_{1 \leq k \leq k_{0}} f\left(u_{k-1}\right) \int_{u_{k-1}}^{u_{k}} \xi(v) d v= \sum_{1 \leq k \leq k_{0}} f\left(u_{k-1}\right) \xi\left(u_{k-1}\right)\left(u_{k}-u_{k-1}\right) \\
&+\sum_{1 \leq k \leq k_{0}} f\left(u_{k-1}\right) \int_{u_{k-1}}^{u_{k}}\left(\xi(v)-\xi\left(u_{k-1}\right)\right) d v \\
& \sum_{1 \leq k \leq k_{0}} f\left(u_{k-1}\right) \xi\left(u_{k-1}\right)\left(u_{k}-u_{k-1}\right) \stackrel{\mathrm{P}}{\rightarrow} \int_{0}^{s} f(u) \xi(u) d u, \quad \alpha \rightarrow 0 .
\end{aligned}
$$


For $C_{0}=\max _{u}|f(u)|$, from (2.1) we get

$$
\begin{aligned}
& \left\|\sum_{1 \leq k \leq k_{0}} f\left(u_{k-1}\right) \int_{u_{k-1}}^{u_{k}}\left(\xi(v)-\xi\left(u_{k-1}\right)\right) d v\right\| \\
& \quad \leq 16 \max _{V}\left\|C_{0} \sum_{k \in V} \int_{u_{k-1}}^{u_{k}}\left(\xi(v)-\xi\left(u_{k-1}\right)\right) d v\right\| \\
& \quad=16 \max _{V}\left\|C_{0}\left(\int_{\bigcup_{k \in V}\left[u_{k-1}, u_{k}\right]} \xi(v) d v-\sum_{k \in V} \xi\left(u_{k-1}\right)\left(u_{k}-u_{k-1}\right)\right)\right\| .
\end{aligned}
$$

By Lemma 3.2, the last value tends to 0 as $\alpha \rightarrow 0$.

Further, we take the obvious equality

$$
\begin{aligned}
f(s) \int_{0}^{s} \xi(v) d v= & \sum_{1 \leq k \leq k_{0}}\left(f\left(u_{k}\right)-f\left(u_{k-1}\right)\right) \int_{0}^{u_{k}} \xi(v) d v \\
& +\sum_{1 \leq k \leq k_{0}} f\left(u_{k-1}\right) \int_{u_{k-1}}^{u_{k}} \xi(v) d v
\end{aligned}
$$

and pass to the limit as $\alpha \rightarrow 0$.

\section{Parabolic equation with a general stochastic measure}

Consider the differential operator

$$
A g(x)=\sum_{1 \leq i, j \leq d} a_{i j}(x) \frac{\partial^{2} g(x)}{\partial x_{i} \partial x_{j}}+\sum_{1 \leq i \leq d} b_{i}(x) \frac{\partial g(x)}{\partial x_{i}}+c(x) g(x),
$$

where

$$
g: \mathbb{R}^{d} \rightarrow \mathbb{R} \quad \text { and } \quad a_{i j}=a_{j i} .
$$

Suppose that $A$ is strongly elliptic in $\mathbb{R}^{d}$ (see (4.5) in [5]).

Assumption 1. All functions

$$
a_{i j}, \quad b_{i}, \quad c, \quad \frac{\partial a_{i j}}{\partial x_{i}}, \quad \frac{\partial^{2} a_{i j}}{\partial x_{i} \partial x_{j}}, \quad \frac{\partial b_{i}}{\partial x_{i}}
$$

are bounded and Hölder continuous in $\mathbb{R}^{d}$.

From now on let $\mu$ be a stochastic measure on Borel subsets of $[0, T]$.

We will study the equation

$$
d X(x, t)=A X(x, t) d t+f(x, t) d \mu(t), \quad X(x, 0)=\xi(x),
$$

where

$$
X: \mathbb{R}^{d} \times[0, T] \rightarrow L_{0}
$$

is an unknown random function.

We consider (1.1) in the weak sense, i.e.

$$
\begin{aligned}
\int_{\mathbb{R}^{d}} X(x, t) \varphi(x) d x= & \int_{\mathbb{R}^{d}} \xi(x) \varphi(x) d x+\int_{\mathbb{R}^{d}} A^{*} \varphi(x) d x \int_{0}^{t} X(x, s) d s \\
& +\int_{[0, t]} d \mu(s) \int_{\mathbb{R}^{d}} f(x, s) \varphi(x) d x
\end{aligned}
$$

for all test functions $\varphi \in \mathcal{S}\left(\mathbb{R}^{d}\right)$ (rapidly decreasing Schwartz functions from $\mathbb{C}^{\infty}\left(\mathbb{R}^{d}\right)$ ). For each fixed $t \in[0, T]$ equality (6.1) holds a.s. Integrals of random functions with 
respect to $d x$ and $d s$ are considered in the Riemann sense (see section $\left[3\right.$ ), and $A^{*}$ denotes the adjoint operator of $A$.

Assumption 2. $\xi: \mathbb{R}^{d} \rightarrow L_{0}$ is such that $\xi(\cdot, \omega)$ is continuous and bounded in $\mathbb{R}^{d}$ for each fixed $\omega \in \Omega$.

Assumption 3. $f: \mathbb{R}^{d} \times[0, T] \rightarrow \mathbb{R}$ is Borel measurable,

$$
\sup _{t}|x|^{-k}|f(x, t)| \rightarrow 0, \quad|x| \rightarrow \infty
$$

for some $k>0$, and $f(x, \cdot)$ is continuous and bounded in $\mathbb{R}^{d}$ for each fixed $t \in[0, T]$.

By Theorem 1, $\S 4$ of [5], under Assumption 1, the equation $\partial g / \partial t=A g$ has a fundamental solution $p(x, y, t-s)$ (recall that coefficients of $A$ do not depend on $t$ ). The following estimate is well known:

$$
|p(x, y, t)| \leq C_{1} t^{-d / 2} \exp \left\{-C_{2}|x-y|^{2} / t\right\}
$$

(see, for example, (4.16) in [5]). Consider the semigroup

$$
S(t) g(x)=\int_{\mathbb{R}^{d}} p(x, y, t) g(y) d y, \quad t>0, S(0) g(x)=g(x) .
$$

Theorem 2, $\S 4$ of [5] implies that for any continuous bounded $g$,

$$
S(t) g(x)=g(x)+A \int_{0}^{t}[S(s) g(x)] d s .
$$

Theorem 6.1. Suppose Assumptions 13 hold. Then the random function

$$
X(x, t)=S(t) \xi(x)+\int_{[0, t]}[S(t-s) f(x, s)] d \mu(s)
$$

is the solution of (6.1).

In addition, suppose that the operator $A$ is self-adjoint and that $X(x, t)$ satisfies (6.1), is integrable on $\mathbb{R}^{d} \times[0, T]$ with respect to $d x \times d t$, is integrable on $\mathbb{R}^{d}$ with respect to $d x$ for each fixed $t$, and is integrable on $[0, T]$ with respect to $d t$ for each fixed $x$. Then $X(x, t)$ is given by (6.3).

Proof. From (6.2) it follows that for $X_{1}(x, t)=S(t) \xi(x)$ and $f=0$ equality (6.1) holds. For

$$
X_{2}(x, t)=\int_{[0, t]}[S(t-s) f(x, s)] d \mu(s)
$$


we have

$$
\begin{aligned}
& \int_{\mathbb{R}^{d}} A^{*} \varphi(x) d x \int_{0}^{t} X_{2}(s) d s+\int_{[0, t]} d \mu(s) \int_{\mathbb{R}^{d}} f(x, s) \varphi(x) d x \\
& =\int_{\mathbb{R}^{d}} A^{*} \varphi(x) d x \int_{0}^{t} d s \int_{[0, s]}[S(s-u) f(x, u)] d \mu(u) \\
& +\int_{[0, t]} d \mu(s) \int_{\mathbb{R}^{d}} f(x, s) \varphi(x) d x \\
& \stackrel{\text { 4.11) }}{=} \int_{\mathbb{R}^{d}} A^{*} \varphi(x) d x \int_{[0, t]} d \mu(u) \int_{u}^{t}[S(s-u) f(x, u)] d s \\
& +\int_{[0, t]} d \mu(s) \int_{\mathbb{R}^{d}} f(x, s) \varphi(x) d x \\
& \stackrel{\text { (4.2) }}{=} \int_{[0, t]} d \mu(u) \int_{\mathbb{R}^{d}} A^{*} \varphi(x) d x \int_{u}^{t}[S(s-u) f(x, u)] d s \\
& +\int_{[0, t]} d \mu(s) \int_{\mathbb{R}^{d}} f(x, s) \varphi(x) d x \\
& =\int_{[0, t]} d \mu(u) \int_{\mathbb{R}^{d}} \varphi(x) d x A \int_{u}^{t}[S(s-u) f(x, u)] d s \\
& +\int_{[0, t]} d \mu(s) \int_{\mathbb{R}^{d}} f(x, s) \varphi(x) d x \\
& \stackrel{\sqrt[6.2]{=}}{=} \int_{[0, t]} d \mu(u) \int_{\mathbb{R}^{d}} \varphi(x) d x([S(t-u) f(x, u)]-f(x, u)) \\
& +\int_{[0, t]} d \mu(s) \int_{\mathbb{R}^{d}} f(x, s) \varphi(x) d x \\
& =\int_{[0, t]} d \mu(u) \int_{\mathbb{R}^{d}} \varphi(x)[S(t-u) f(x, u)] d x \\
& \stackrel{\text { (4.2) }}{=} \int_{\mathbb{R}^{d}} \varphi(x) d x \int_{[0, t]}[S(t-s) f(x, s)] d \mu(s) \\
& =\int_{\mathbb{R}^{d}} X_{2}(x, t) \varphi(x) d x .
\end{aligned}
$$

Therefore (6.1) holds for $X=X_{1}+X_{2}$.

Finally, we will prove the uniqueness of the solution. Section 4 implies that the random function $X$ given by (6.3) is integrable. Thus, it is enough to prove that the equation

$$
\int_{\mathbb{R}^{d}} X(x, t) \varphi(x) d x=\int_{\mathbb{R}^{d}} A^{*} \varphi(x) d x \int_{0}^{t} X(x, s) d s
$$

has only the zero solution provided that $A=A^{*}$.

For $\varphi \in \mathcal{S}\left(\mathbb{R}^{d}\right)$ and $0<s<t$ set

$$
\psi_{t, s}(x)=S(t-s) \varphi(x)
$$

Then

$$
\psi_{t, s} \in \mathcal{S}\left(\mathbb{R}^{d}\right), \quad A \psi_{t, s}+\frac{\partial}{\partial s} \psi_{t, s}=0, \quad \psi_{t, s} \rightarrow \varphi
$$


uniformly on any bounded subset of $\mathbb{R}^{d}$ as $t \downarrow s$ (see (4.13) in [5]), and we get

$$
\begin{array}{rl}
\int_{\mathbb{R}^{d}} & X(x, t) \psi_{t, s}(x) d x \stackrel{\text { (6.4) }}{=} \int_{\mathbb{R}^{d}} A \psi_{t, s}(x) d x \int_{0}^{s} X(x, u) d u \\
& \stackrel{(5.6)}{=} \int_{\mathbb{R}^{d}} d x \int_{0}^{s} A \psi_{t, u}(x) X(x, u) d u+\int_{\mathbb{R}^{d}} d x \int_{0}^{s} A \frac{\partial}{\partial u} \psi_{t, u}(x) d u \int_{0}^{u} X(x, v) d v \\
& \stackrel{(4.5)}{=} \int_{\mathbb{R}^{d}} d x \int_{0}^{s} A \psi_{t, u}(x) X(x, u) d u+\int_{0}^{s} d u \int_{\mathbb{R}^{d}} A \frac{\partial}{\partial u} \psi_{t, u}(x) d x \int_{0}^{s} X(x, v) d v \\
& \stackrel{(6.4)}{=} \int_{\mathbb{R}^{d}} d x \int_{0}^{s} A \psi_{t, u}(x) X(x, u) d u+\int_{0}^{s} d u \int_{\mathbb{R}^{d}} \frac{\partial}{\partial u} \psi_{t, u}(x) X(x, u) d x \\
& \stackrel{(4.5)}{=} \int_{\mathbb{R}^{d}} d x \int_{0}^{s}\left(A \psi_{t, u}(x)+\frac{\partial}{\partial u} \psi_{t, u}(x)\right) X(x, u) d u=0 .
\end{array}
$$

Passing to the limit as $t \downarrow s$ and applying Lemma 3.9. we arrive at

$$
\int_{\mathbb{R}^{d}} X(x, s) \varphi(x) d x=0 .
$$

Example 6.1. Let the stochastic measure $\mu$ be generated by a continuous square integrable martingale $Y, \mu(\mathrm{A})=\int_{0}^{T} \mathbf{1}_{\mathrm{A}}(t) d Y(t)$, and $\lambda$ be the Lebesgue measure on $\mathbb{R}^{d}$. Then

$$
M_{t}(\mathrm{~A})=Y(t) \lambda(\mathrm{A}), \quad 0 \leq t \leq T, \mathrm{~A} \subset \mathbb{R}^{d},
$$

is a worthy martingale measure with the dominating measure

$$
K(\mathrm{~A} \times \mathrm{B} \times(0, t])=\left|\langle Y\rangle_{t}\right| \lambda(\mathrm{A}) \lambda(\mathrm{B})
$$

(we use the terminology of [19]). In this case, (6.3) leads to

$$
\begin{aligned}
X(x, t) & =\int_{\mathbb{R}^{d}} p(x, y, t) \xi(y) d y+\int_{[0, t]} d \mu(s) \int_{\mathbb{R}^{d}} p(x, y, t-s) f(y, s) d y \\
& =\int_{\mathbb{R}^{d}} p(x, y, t) \xi(y) d y+\int_{[0, t] \times \mathbb{R}^{d}} p(x, y, t-s) f(y, s) M(d y d s) .
\end{aligned}
$$

The results of [19, Chapter 2] imply that the integral with respect to $M(d y d s)$ is well defined and is the limit of integrals of elementary functions. For an elementary function, the equality of two stochastic integrals in (6.5) is obvious. Further, we can use the dominated convergence theorem for the integral with respect to $d \mu(s)$.

A similar solution of the parabolic SPDE with respect to general martingale measure is given in Example 9 and Remark 20 of [3].

Example 6.2. Assume that $\mu$ is generated by the real-valued Wiener process $w$ and $\mathcal{J}$ denotes the set of Schwartz rapidly decreasing test functions in $\mathbb{R}^{d}$. Then the equation

$$
\langle\mathcal{W}(t), \psi\rangle=w(t) \int_{\mathbb{R}^{d}} \psi(x) d x, \quad \psi \in \mathcal{J},
$$

defines the spatially homogeneous Wiener process with values in $\mathcal{J}^{\prime}$ (we used the terminology of [10]). For this case, our equality (6.3) is a partial case of the mild solution (2.6) in $[10]$.

Remark. In a similar manner, we can consider a more general equation

$$
d X(x, t)=A X(x, t) d t+\sum_{1 \leq i \leq j} f_{i}(x, t) d \mu_{i}(t), \quad X(0)=\xi,
$$

which includes the case

$$
d X(x, t)=A X(x, t) d t+f_{1}(x, t) d t+f_{2}(x, t) d \mu(t), \quad X(0)=\xi .
$$


The solution of (6.6) is

$$
X(x, t)=S(t) \xi(x)+\sum_{1 \leq i \leq j} \int_{[0, t]}\left[S(t-s) f_{i}(x, s)\right] d \mu_{i}(s) .
$$

Under assumptions of Theorem 6.1] the solution of (6.6) is unique.

\section{BIBLIOGRAPHY}

1. S. Albeverio, J.-L. Wua, and T.-S. Zhang, Parabolic SPDEs driven by Poisson white noise, Stochastic Process. Appl. 74 (1998), 21-36. MR1624076 (99c:60124)

2. G. P. Curbera and O. Delgado, Optimal domains for $L_{0}$-valued operators via stochastic measures, Positivity 11 (2007), 399-416. MR2336205 (2008g:46063)

3. R. C. Dalang, Extending martingale measure stochastic integral with applications to spatially homogeneous s.p.d.e's, Electron. J. Probab. 4 (1999), 1-29. MR.1684157 (2000b:60132)

4. G. Da Prato and J. Zabczyk, Stochastic equations in infinite dimensions, Encyclopedia Math. Appl., vol. 44, Cambridge Univ. Press, Cambridge, 1992. MR.1207136 (95g:60073)

5. A. M. Ilyin, A. S. Kalashnikov, and O. A. Oleynik, Linear second-order partial differential equations of the parabolic type, J. Math. Sci. (N. Y.) 108 (2002), 435-542. MR.1875963(2003a:35087)

6. P. Kotelenez, Stochastic Ordinary and Stochastic Partial Differential Equations: Transition from Microscopic to Macroscopic Equations, Stochastic Modelling Appl. Probab., vol. 58, Springer-Verlag, Berlin-Heidelberg-New York, 2007. MR2370567 (2009h:60004)

7. S. Kwapień and W. A. Woycziński, Random Series and Stochastic Integrals: Single and Multiple, Birkhäuser, Boston, 1992. MR1167198 (94k:60074)

8. J. Memin, Yu. Mishura, and E. Valkeila, Inequalities for the moments of Wiener integrals with respect to a fractional Brownian motion, Statistics and Probability Letters 27 (2001), 197-206. MR:1822771 (2002b:60096)

9. S. M. Nikolsky, A Course of Mathematical Analysis, vol. 2, "Mir", Moscow, 1977. MR0466436 $(57: 6315 \mathrm{~b})$

10. S. Peszat and J. Zabczyk, Stochastic evolution equations with a spatially homogeneous Wiener process, Stochastic Process. Appl. 72 (1997), 187-204. MR.1486552 (99k:60166)

11. S. Peszat and J. Zabczyk, Stochastic partial differential equations with Lévy noise: an evolution equation approach, Encyclopedia Math. Appl., vol. 113, Cambridge Univ. Press, Cambridge, 2007. MR2356959 (2009b:60200)

12. V. Radchenko, Integrals with Respect to General Stochastic Measures, Institute of Mathematics, Kyiv, 1999. (Russian)

13. V. Radchenko, Heat equation and wave equation with general stochastic measures, Ukrain. Mat. Zh. 60 (2008), 1675-1685; English transl. in Ukrainian Math. J. 60 (2008), 1968-1981. MR2523115(2010d:60117)

14. V. Radchenko, Mild solution of the heat equation with a general stochastic measure, Studia Math. 194 (2009), 231-251. MR2539554(2010j:60157)

15. S. Rolewicz, Metric Linear Spaces, Monografie Matematyczne, vol. 56, PWN-Polish Scientific Publishers, Warsaw, 1972. MR0438074 (55:10993)

16. C. Ryll-Nardzewski and W. A. Woyczyński, Bounded multiplier convergence in measure of random vector series, Proc. Amer. Math. Soc. 53 (1975), 96-98. MR0385960 (52:6819)

17. M. Talagrand, Les mesures vectorielles à valeurs dans $L_{0}$ sont bornées, Ann. Sci. École Norm. Sup. (4) 14 (1981), 445-452. MR654206 (83f:28007)

18. Ph. Turpin, Convexités dans les espaces vectoriels topologiques généraux, Dissertationes Math. 131 (1976). MR0423044 (54:11028)

19. J. B. Walsh, An introduction to stochastic partial differential equations, Lect. Not. Math. 1180 (1984), 236-434.

Department of Mathematical Analysis, Kyiv national Taras Shevchenko University, Kyiv 01601, UKRAINE

E-mail address: vradchenko@univ.kiev.ua

Received 22/DEC/2011

Originally published in English 